Document downloaded from:

http://hdl.handle.net/10251/56594

This paper must be cited as:

Jiao, L.; Li, FY.; Pla, V. (2012). Modeling and Performance Analysis of Channel Assembling in Multi-channel Cognitive Radio Networks with Spectrum Adaptation. IEEE Transactions on Vehicular Technology. 61:2686-2697. doi:10.1109/TVT.2012.2196300.

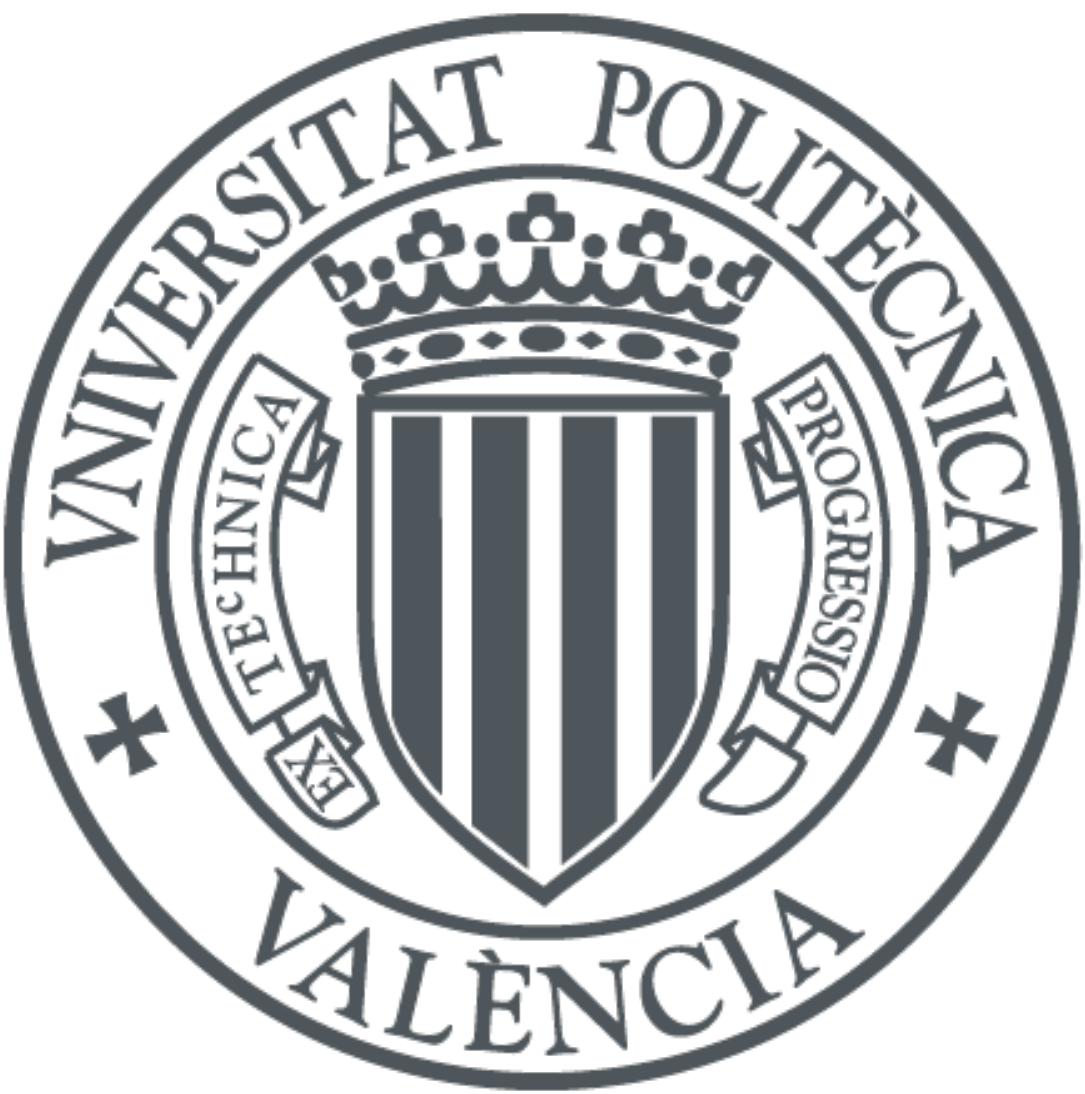

The final publication is available at

http://dx.doi.org/10.1109/TVT.2012.2196300

Copyright Institute of Electrical and Electronics Engineers (IEEE)

Additional Information 


\title{
Modeling and Performance Analysis of Channel Assembling in Multi-channel Cognitive Radio Networks with Spectrum Adaptation
}

\author{
Lei Jiao, Student Member, IEEE, Frank Y. Li, Senior Member, IEEE, and Vicent Pla
}

\begin{abstract}
To accommodate spectrum access in multi-channel cognitive radio networks, channel assembling technique which combines several channels together as one channel has been proposed in many MAC protocols. However, analytical models for cognitive radio networks enabled with this technique have not been thoroughly investigated so far. In this paper, two representative channel assembling strategies are proposed considering spectrum adaptation and heterogeneous traffic, and the performance of these strategies is evaluated based on proposed continuous time Markov chain models. Moreover, approximations of these models in the quasi-stationary regime are analyzed and closedform capacity expressions are derived in different conditions. The performance of different strategies including the one without assembling is compared with each other based on the numerical results obtained from these models and validated by extensive simulations. Furthermore, simulation studies are also performed for other types of traffic distributions in order to evaluate the validity and the preciseness of the mathematical models. Through both analyses and simulations, we demonstrate that channel assembling represented by those investigated strategies can improve system performance if a proper strategy is selected with appropriate system parameter configurations.
\end{abstract}

Index Terms-Cognitive radio networks, channel assembling, spectrum adaptation, continuous time Markov chain models, performance analysis.

\section{INTRODUCTION}

C ONSIDER typical scenarios in cognitive radio networks (CRNs) [1] where multiple channels exist. There are two options for channel access. On the one hand, secondary users (SUs) can follow the original channel structure for primary users (PUs), as proposed in many CRN MAC designs [2][5]. On the other hand, with the support of more sophisticated hardware, SUs can make a decision on whether to assemble multiple idle channels together as one channel for providing higher data rate or still treat them as separated channels. In the latter case, when two or more channels in the frequency

Manuscript received 11 September 2011, revised 1 February 2012, accepted 30 March 2012.

Copyright (c) 2012 IEEE. Personal use of this material is permitted. However, permission to use this material for any other purposes must be obtained from the IEEE by sending a request to pubs-permissions@ieee.org.

Lei Jiao and Frank Y. Li are with the Dept. of Information and Communication Technology, University of Agder (UiA), N-4898 Grimstad, Norway. E-mail: \{lei.jiao; frank.li\}@uia.no.

Vicent Pla is with the Dept. of Communications, Universidad Politècnica de València (UPV), 46022 València, Spain. E-mail: vpla@dcom.upv.es.

This research is supported by the EU Seventh Framework Programme FP7-PEOPLE-IRSES under grant agreement no. 247083, project acronym S2EuNet, and by the Spanish government through project TIN2010-21378$\mathrm{C} 02-02$. domain are idle, they can be combined together as one channel using channel assembling. This technique has indeed been utilized in many CRN MAC protocols [6]-[11].

The research work on CRN spectrum access can be categorized into two phases. The first phase is MAC protocol design itself, aiming at proposing feasible strategies in order to allow CRNs access spectrum in a more efficient way [2][11]. The second phase is to build analytical models in order to help us better understand the theory behind these protocols and evaluate the performance of different strategies [12]-[17]. In this study, we aim at the second phase and focus on the performance analysis of channel assembling. This work is motivated by the observation that although widely discussed in CRN MAC protocols, the performance of CRNs with channel assembling itself has not been thoroughly analyzed through recognized mathematical models. For example, the performance of an SU network when a PU channel could be divided into several SU channels is analyzed based on a continuous time Markov chain (CTMC) model in [13]-[15]. In our previous work [16], the performance of several channel assembling strategies when spectrum adaptation is not enabled is studied through CTMC models. In a similar study [17], channel aggregation for CRNs is analyzed mathematically with and without spectrum handover. However, only homogeneous traffic is considered in CRNs in all those studies, and none of them analyze channel assembling strategies with spectrum adaptation systematically through mathematical modeling.

The meanings of spectrum adaptation are twofolds. On the one hand, it is inherited from spectrum handover, which allows SUs to switch an ongoing SU transmission to a vacant channel that is not occupied by PUs or SUs, if it exists, when a PU activity appears on the current channel. On the other hand, it is meant that an ongoing SU service can adaptively adjust the number of assembled channels according to the availability of channels as well as other SUs' activities. How spectrum adaptation is utilized depends on specific channel assembling strategies. Furthermore, spectrum adaptation can be performed either by a base station centrally or by the cognitive radios themselves through a suitable distributed protocol. Since spectrum adaptation is one of the essential principles of CRNs, i.e., sensing the radio environment and adapting to it accordingly, we propose and investigate two representative channel assembling strategies with spectrum adaptation in this study. Based on these proposed strategies, we present CTMC models to analyze their performance. Then, the models in the quasi-stationary regime (QSR), i.e., when the time scale of 
PU activities is much larger than that of the SU activities, are introduced. Although various assembling strategies exist in different systems, similar modeling process can be developed, and we believe that, with minor modifications, the models we develop for these two strategies can also be applied to many other strategies. Thirdly, numerical results obtained from mathematical models are analyzed and compared, and the correctness of the mathematical models is validated by extensive simulations. Finally, to evaluate the preciseness and the robustness of the mathematical models, the results under traffic distributions rather than the ones used in CTMC models are examined by simulations and compared with the analytical results from the CTMC models.

In brief, the major contributions of this paper can be outlined as follows:

- Two representative channel assembling strategies for CRNs considering heterogeneous traffic are proposed and thoroughly investigated. Although the strategies may be complex from implementation point of view, the analyses of them still have significance as they provide theoretical insight on the performance gain that can be obtained by channel assembling.

- Mathematical models for analyzing these strategies are proposed using CTMCs. This allows us to examine the performance of those strategies theoretically by assuming Poisson traffic arrival processes and exponential service time distributions for PUs and SUs.

- The approximations for these models in the QSR are introduced. With these approximations, closed-form expressions for capacity calculation are derived for different types of traffic.

- Other more realistic traffic patterns which may not follow the Poisson arrival and the exponential service time assumptions are evaluated through extensive simulations. Therefore, the rationality and the robustness as well as the applicability of the proposed mathematical models are verified to a much larger extent.

The rest of this paper is organized as follows. Section II summarizes the related work. The system model and assumptions are given in Section III. After the channel assembling strategies are described in Section IV, different CTMC models are built in Section V in order to analyze the performance of these strategies. Furthermore, the models of these strategies in the QSR is analyzed in Section VI, followed by numerical results and corresponding discussions presented in Section VII. Finally, the paper is concluded in Section VIII.

\section{RELATED WORK}

In this section, we summarize the state-of-the-art CTMC models established for different channel access strategies and application scenarios in CRNs.

In [13], [14] and [15], the case when a PU channel could be split into several SU channels are modeled through CTMCs. The authors of [13] and [14] mainly focus on the cases when there are infinite number of users, and both spectrum handover and non-handover cases are considered. In [15], it is analyzed the performance of the secondary network in the cases with a finite user population. In [12], three spectrum access schemes are proposed and analyzed through CTMCs. However, channel assembling is not considered in that paper.

In [16] and [17], channel assembling is investigated mathematically from different angles. In [16], the performance of the secondary network with different channel assembling strategies is studied when spectrum adaptation is not implemented. Three strategies, i.e., without channel assembling, with a fixed number of assembled channels, and assembling all idle channels when SU services access the system, are investigated and compared. Similarly, in [17], three channel assembling strategies with and without spectrum handover are studied. However, the CTMC models and some parameters used in [17] are not appropriately adjusted. Moreover, the second meaning of spectrum adaptation, i.e., the number of assembled channels for ongoing SU services can be adapted, is not considered in these studies, neither in [16] nor in [17]. In our recent work [18], different channel assembling strategies with spectrum adaptation are studied. Based on these preliminary studies, we propose two representative channel assembling strategies considering spectrum adaptation and heterogeneous traffic, and investigate them in depth afterwards.

\section{SYSTEM MODEL AND ASSUMPTIONS}

Before we describe channel assembling strategies, the system model and the assumptions are given as follows. Assume that there are two types of radios, PUs and SUs, operating in the same frequency band which consists of $M \in \mathbb{N}^{+}$channels for PUs, where $\mathbb{N}^{+}$denotes the set of positive natural numbers. PUs have priority to utilize the spectrum and can acquire the channels being used by SUs at any time. Each PU service occupies only one channel while SUs can assemble multiple channels. Those multiple channels can be either neighboring to or separated from one another in the spectrum domain.

In this work, we consider two heterogeneous SU traffic types, i.e., elastic traffic and real time traffic. For elastic traffic, like file downloading, the service time will be reduced if more channels are utilized for the same service due to higher data rate. On the other hand, for a real time traffic flow, like a real time voice conversation, sufficient service quality is provided given a fixed number of channels, and its service time is fixed even if more channels are assembled ${ }^{1}$. Since channel assembling is more beneficial for elastic traffic because of its flexibility, our focus will be mainly on the elastic traffic in this analysis. An admission control phase which can provide further tradeoff between both types of SU services can be applied according to the calculated blocking probability based our models of various strategies. Once accepted, the SU services will experience stable services without interruption due to channel fading with the help of advanced underlined physical layer techniques.

For the sake of conciseness, we refer to traffic flows as services, and use events to indicate the arrival and departure of services for both PUs and SUs. In the following analysis, we focus on the performance of the secondary network. It

\footnotetext{
${ }^{1}$ Of course the quality of the voice conversation will be improved up to a limit when more channels are assembled.
} 
is assumed that the services of the secondary network are independent of each other. PUs are not aware of the existence of SU activities, and SUs can detect PU activities with high enough spectrum sensing accuracy. We further assume that the sensing and spectrum adaptation latency is much shorter than the duration of the service events. In other words, the arrivals or departures of services are unlikely to happen during the sensing and spectrum adaptation period. Furthermore, it is assumed that there is a protocol running among SUs to support channel assembling and spectrum adaptation.

\section{Channel ASSEMBling STRATEGIES}

To study SU performance with channel assembling, two strategies with spectrum adaptation are proposed, as presented below. For the first strategy, i.e., the $\operatorname{Static}(a, W, V)$ strategy, referred to as $S(a, W, V)$, the first meaning of spectrum adaptation, i.e., spectrum handover only, is applied. The second strategy, Dynamic $(a, W, V)$, referred to as $D(a, W, V)$, uses the full meaning of spectrum adaption, i.e., not only the spectrum handover is adopted, but also the number of the assembled channels for an ongoing SU service is adjustable. In our notations, parameter $a \in \mathbb{N}^{+}$indicates the number of channels assembled by a real time SU service in the network. Parameters $W, V \in \mathbb{N}^{+}$represent, respectively, the lower bound and the upper bound of the number of assembled channels for an elastic SU service. The values of them are predefined for a given strategy and network configuration, at the network initiation phase. Let $N \in \mathbb{N}^{+}, W \leq N \leq V$, denote the number of channels that an elastic SU service assembles, which may vary from one elastic SU service to another, and may also vary along time for an ongoing elastic SU service when the dynamic strategy is employed. However, the number of channels for a real time service is always fixed. Denote a real time SU service and an elastic SU service by $\mathrm{SU}_{r}$ and $\mathrm{SU}_{e}$ respectively.

\section{A. $S(a, W, V)$}

In this strategy, upon an $\mathrm{SU}_{r}$ arrival, the service can commence if the number of idle channels is larger than or equal to $a$. When an $\mathrm{SU}_{e}$ tries to access channels, if the number of idle channels, denoted by $I_{c}$, is larger than or equal to $W$ at this moment, it can commence with the number of assembled channels as $\min \left\{V, I_{c}\right\}$. If not enough idle channels exist, i.e., the number of idle channels is lower than $W$ for an $\mathrm{SU}_{e}$ or $a$ for an $\mathrm{SU}_{r}$, the service will be blocked. Static here means that once an SU service starts, the number of assembled channels cannot be changed any longer. In other words, during an SU service time period, even if new idle channels emerge, for instance when any PUs or other SUs finish their transmissions, ongoing SU services will not assemble those newly available channels. On the other hand, when an arriving PU takes any one of the assembled channels that is in use by an ongoing SU service, the SU service will switch to an idle channel which is not occupied by PUs or SUs, if it exists. Otherwise, the SU service is forced to terminate.

A special case of this strategy is $a=W=V=1$, which represents the legacy channel access strategy without channel assembling. We denote it as no assembling $(N A)$ and its performance will be compared with the proposed two strategies later in this paper.

\section{B. $D(a, W, V)$}

If there are enough idle channels upon an SU arrival, i.e., the number is larger than or equal to $W$ for an $\mathrm{SU}_{e}$ or $a$ for an $\mathrm{SU}_{r}$, it will react in the same way as in the static strategy. However, if there are not enough idle channels for a newly arrived SU service, instead of blocking it, ongoing $\mathrm{SU}_{e} \mathrm{~s}$ will share their occupied channels to the newly arrived SU service, as long as they can still keep at least $W$ channels and the number is sufficient for the new SU service to commence after sharing. When an SU service needs the channels shared by ongoing $\mathrm{SU}_{e} \mathrm{~S}$ to commence and there are several $\mathrm{SU}_{e} \mathrm{~S}$ in the system, the one that occupies the maximum number of channels will donate first, due to the consideration of fairness among SU services. If the $\mathrm{SU}_{e}$ with the maximum number cannot provide enough channels, the one with the second maximum number will share its channels then, and so on. The newly arrived $\mathrm{SU}_{e}$ will join the network as long as $W$ channels are gathered, including both the idle channels and the ones released by other $\mathrm{SU}_{e} \mathrm{~s}$, if the number of idle channels is lower than $W$. If the number of idle channels together with the number of channels to be released by all $\mathrm{SU}_{e} \mathrm{~s}$ is still not enough, i.e., the number is lower than $W$ for an $\mathrm{SU}_{e}$ or $a$ for an $\mathrm{SU}_{r}$, the new $\mathrm{SU}$ arrival is blocked.

In this strategy, if any channels become idle, the ongoing $\mathrm{SU}_{e} \mathrm{~s}$ with less than $V$ channels will assemble those newly available channels up to $V$. In the presence of multiple $\mathrm{SU}_{e} \mathrm{~s}$ that can utilize newly vacant channels, it designates the one which currently has the minimum number of channels to occupy them first, again, due to the fairness consideration mentioned above. If the $\mathrm{SU}_{e}$ with the minimum number of channels assembles $V$ channels after adjustment and there are still vacant channels, other $\mathrm{SU}_{e} \mathrm{~s}$ will occupy the remaining ones according to the same principle, until all those vacant channels are utilized or all existing $\mathrm{SU}_{e} \mathrm{~s}$ have $V$ channels.

On the other hand, when PU services arrive and there is no idle channel available, $\mathrm{SU}_{e} \mathrm{~s}$ can flexibly adjust downwards the number of assembled channels, as long as the remaining number of channels is still not fewer than $W$. If an arriving PU acquires any one of the channels that is occupied by an $\mathrm{SU}_{e}$ with exactly $W$ channels and there is no idle channel, this already commenced $\mathrm{SU}_{e}$ is forced to terminate. To give higher priority to $\mathrm{SU}_{r}$, if any one of the channels being used by an $\mathrm{SU}_{r}$ is taken by an arriving PU and there is no idle channel at the moment, the $\mathrm{SU}_{e}$ with the maximum number of channels will donate one channel from its occupied set. In this way, the $\mathrm{SU}_{r}$ can be kept. A forced $\mathrm{SU}_{r}$ termination happens only if it is interrupted by a $\mathrm{PU}$ arrival when all $\mathrm{SU}_{e} \mathrm{~S}$ occupy exactly $W$ channels and no idle channel exists.

\section{CTMC MODELS FOR CHANNEL ASSEMBLING STRATEGIES}

To model different strategies with channel assembling, we develop CTMCs by assuming that the arrivals of both SU and 
TABLE I

TRANSITIONS FROM A GENERIC STATE $\boldsymbol{x}=\left(i, g_{a}, j_{W}, \ldots, j_{V}\right)$ OF $S(a, W, V)$.

\begin{tabular}{llll}
\hline Activity & Destination state & Trans. rate & Conditions \\
\hline PU DP ${ }^{2}$. & $\left(i-1, g_{a}, j_{W}, \ldots, j_{V}\right)$ & $i \mu_{P}$ & $i>0$. \\
PU AR, no FT. & $\left(i+1, g_{a}, j_{W}, \ldots, j_{V}\right)$ & $\lambda_{P}$ & $b(\boldsymbol{x})<M$. \\
PU AR, FT in an $\mathrm{SU}_{r}$. & $\left(i+1, g_{a}-1, j_{W}, \ldots, j_{V}\right)$ & $\frac{a g_{a}}{M-i} \lambda_{P}$ & $b(\boldsymbol{x})=M ; i<M ; g_{a}>0$. \\
& & $\frac{k j_{k}}{M-i} \lambda_{P}$ & $b(\boldsymbol{x})=M ; i<M ; j_{k}>0 ; W \leq k \leq V$. \\
PU AR, FT in an $\mathrm{SU}_{e}(k)$. & $\left(i+1, g_{a}, j_{W}, \ldots, j_{k}-1, \ldots, j_{V}\right)$ & $g_{a} \mu_{S}^{\prime}$ & $g_{a}>0$. \\
$\mathrm{SU}_{r}$ DP. & $\left(i, g_{a}-1, j_{W}, \ldots, j_{V}\right)$ & $k j_{k} \mu_{S}$ & $j_{k}>0 ; W \leq k \leq V$. \\
$\mathrm{SU}_{e}(k)$ DP. & $\left(i, g_{a}, j_{W}, \ldots, j_{k}-1, \ldots, j_{V}\right)$ & $\lambda_{S}^{\prime}$ & $M-b(\boldsymbol{x}) \geq a$. \\
$\mathrm{SU}_{r}$ AR. & $\left(i, g_{a}+1, j_{W}, \ldots, j_{V}\right)$ & $\lambda_{S}$ & $k=\min \{V, M-b(\boldsymbol{x})\} \geq W$. \\
$\mathrm{SU}_{e}$ AR. & $\left(i, g_{a}, j_{W}, \ldots, j_{k}+1, \ldots, j_{V}\right)$ & & \\
\hline
\end{tabular}

PU services are Poisson processes. The SU service arrival rates are $\lambda_{S}$ and $\lambda_{S}^{\prime}$ for elastic and real time services respectively, and the PU service arrival rate is given by $\lambda_{P}$. Upon a PU service arrival, it will access one of the channels that are not occupied by ongoing PU services with the same probability. Similarly, the service times are exponentially distributed. The service rates of PU services and SU real time services are $\mu_{P}$ and $\mu_{S}^{\prime}$ respectively, and the service rate for elastic SU services is $\mu_{S}$ in one channel. For real time traffic, the service rate is constant as stated earlier. For elastic traffic, the service rate will be higher if several channels are accumulated for the same service. Assume further that all the channels are homogeneous. Then the average data rate is approximately linearly increasing with the number of assembled channels. Therefore, the service rate of the $N$ assembled channels for an SU elastic service is approximated as $N$ times of that in one channel, i.e., $N \mu_{S}$. The unit for these parameters could be services/unit time. Given concrete values for these services, the system capacity could be expressed in kbps or Mbps. For this reason, in our analysis and results illustrated later, the unit of system capacity is not explicitly expressed.

According to the above strategies, different CTMC models can be built ${ }^{3}$. The states of these CTMC models can be represented by $\boldsymbol{x}=\left(i, g_{a}, j_{W}, \ldots j_{k}, \ldots, j_{V}\right)$, where $i$ is the number of PU services, $g_{a}$ is the number of $\mathrm{SU}_{r}$ s assembling $a$ channels, while $j_{k}$ is the number of $\mathrm{SU}_{e} \mathrm{~s}$ that assemble $k=$ $W, W+1, \ldots, V$ channels. Denote by $b(\boldsymbol{x})$ the total number of utilized channels at state $\boldsymbol{x}$, as $b(\boldsymbol{x})=i+a g_{a}+\sum_{k=W}^{V} k j_{k}$. We start from modeling the static strategy first.

\section{A. CTMC analysis for $S(a, W, V)$}

Let $\mathcal{S}$ be the set of feasible states, as $\mathcal{S}=$ $\left\{\boldsymbol{x} \mid i, g_{a}, j_{W}, \ldots, j_{V} \geq 0 ; b(\boldsymbol{x}) \leq M\right\}$. Table I summarizes the state transitions in this strategy. Given feasible states and their transitions in a CTMC, we can construct the global balance equations and the normalization equation. That is, the equations which indicate that, in steady-state, for each state the average incoming transition rate is equal to the outgoing

\footnotetext{
${ }^{2}$ For illustration convenience, we use AR to indicate an arrival event, DP to indicate a departure event, and FT to indicate a forced termination event in Table I. Notation $\mathrm{SU}_{e}(k)$ represents an $\mathrm{SU}_{e}$ with $k$ channels in this table. Similar notations apply to Tables II, III, and IV as well.

${ }^{3}$ The protocol overhead for each strategy is different, e.g., the dynamic strategy requires highest control overhead in order to support access flexibility. To keep our analysis simple and consistent for all strategies, the protocol overhead is not included in our models presented below.
}

rate, and that the sum of the probabilities of all states is equal to one. From these equations, the state possibilities, denoted by $\pi(\boldsymbol{x})$ as the probability of state $\boldsymbol{x}$, are obtained. Given the state probabilities $\pi(\boldsymbol{x})$, system performance parameters can be developed as follows.

The blocking probability of a type of SU services is the sum of the probabilities of states that cannot accommodate more SU services of that type. Let $P_{b}$ and $P_{b}^{\prime}$ be the blocking probability of $\mathrm{SU}_{e}$ and $\mathrm{SU}_{r}$ respectively, expressed by

$$
P_{b}=\sum_{\substack{\boldsymbol{x} \in \mathcal{S}, M-b(\boldsymbol{x})<W}} \pi(\boldsymbol{x}) ; \quad P_{b}^{\prime}=\sum_{\substack{\boldsymbol{x} \in \mathcal{S}, M-b(\boldsymbol{x})<a}} \pi(\boldsymbol{x}) .
$$

The capacity of a type of SU services is defined as the average service rate of SUs in that type, i.e., the average number of SU service completions per time unit [13]. Let $\rho$ and $\rho^{\prime}$ be the capacity of $\mathrm{SU}_{e}$ and $\mathrm{SU}_{r}$ respectively, shown as follows,

$$
\rho=\sum_{\boldsymbol{x} \in \mathcal{S}} \sum_{k=W}^{V} k j_{k} \mu_{S} \pi(\boldsymbol{x}) ; \quad \rho^{\prime}=\sum_{\boldsymbol{x} \in \mathcal{S}} g_{a} \mu_{S}^{\prime} \pi(\boldsymbol{x}) .
$$

Forced termination represents a preemption of an ongoing SU service. In this work, we consider the fraction of forced terminations over commenced SU services. Therefore, the forced termination probability can be expressed as the mean forced termination rate (denoted by $R_{f}$ for $\mathrm{SU}_{e}$ and $R_{f}^{\prime}$ for $\mathrm{SU}_{r}$ ) divided by the rate of the commenced $\mathrm{SU}$ services [14] (denoted by $\lambda_{S}^{*}$ for $\mathrm{SU}_{e}$ and $\lambda_{S}^{\prime *}$ for $\mathrm{SU}_{r}$ ). Let $P_{f}$ and $P_{f}^{\prime}$ be the forced termination probability of $\mathrm{SU}_{e}$ and $\mathrm{SU}_{r}$ respectively. They are obtained as follows.

$$
\begin{aligned}
& P_{f}=\frac{R_{f}}{\lambda_{S}^{*}}=\frac{\lambda_{P}}{\lambda_{S}^{*}} \sum_{\substack{\boldsymbol{x} \in \mathcal{S}, b(\boldsymbol{x})=M, i<M}} \sum_{k=W}^{V} \frac{k j_{k}}{b(\boldsymbol{x})-i} \pi(\boldsymbol{x}) ; \\
& P_{f}^{\prime}=\frac{R_{f}^{\prime}}{\lambda_{S}^{\prime *}}=\frac{\lambda_{P}}{\lambda_{S}^{\prime *}} \sum_{\substack{\boldsymbol{x} \in \mathcal{S}, b(\boldsymbol{x})=M, i<M}} \frac{a g_{a}}{b(\boldsymbol{x})-i} \pi(\boldsymbol{x}),
\end{aligned}
$$

where $\lambda_{S}^{*}=\left(1-P_{b}\right) \lambda_{S}$ and $\lambda_{S}^{\prime *}=\left(1-P_{b}^{\prime}\right) \lambda_{S}^{\prime}$.

The average service rate per ongoing SU service, i.e., at which rate an ongoing SU service is processed on average, is defined as the capacity divided by the average number of ongoing SU services of the same type. Let $\mu_{p s}$ and $\mu_{p s}^{\prime}$ be the average service rate per ongoing $\mathrm{SU}$ service of $\mathrm{SU}_{e}$ and $\mathrm{SU}_{r}$ 
TABLE II

TRANSITIONS FROM A GENERIC STATE $\boldsymbol{x}=\left(i, g_{a}, j_{W}, \ldots, j_{k}, \ldots, j_{V}\right)$ OF $D(a, W, V), W \leq k \leq V$ UPON A SERVICE DEPARTURE FOR BOTH PU AND SU SERVICES.

\begin{tabular}{|c|c|c|c|}
\hline Activity & Dest. state & Trans. rate & Conditions \\
\hline PU DP. An $\mathrm{SU}_{e}(k)$ uses the vacant channel. & $\begin{array}{l}\left(i-1, g_{a}, j_{W}, \ldots, j_{k}-1\right. \\
\left.j_{k+1}+1, \ldots, j_{V}\right)\end{array}$ & $i \mu_{P}$ & $\begin{array}{l}j_{k}>0, k=\min \left\{r \mid j_{r}>0, W \leq r \leq V-1\right\} ; i>0 \\
V>1\end{array}$ \\
\hline PU DP. $\mathrm{SU}_{e} \mathrm{~s}$ cannot use the vacant channel. & $\begin{array}{l}\left(i-1, g_{a}, j_{W}, \ldots, j_{k}\right. \\
\left.\ldots, j_{V}\right)\end{array}$ & $i \mu_{P}$ & $j_{k}=0, W \leq k<V ; i>0$. Or $W=V ; i>0$ \\
\hline $\begin{array}{l}\mathrm{SU}_{e}(\mathrm{k}) \text { DP. Other } \mathrm{SU}_{e} \mathrm{~s} \text {, if exist, cannot use the } \\
\text { vacant channel(s). }\end{array}$ & $\begin{array}{l}\left(i, g_{a}, j_{W}, \ldots, j_{k}-1,\right. \\
\left.\ldots, j_{V}\right)\end{array}$ & $k j_{k} \mu_{S}$ & $\begin{array}{l}j_{k}=1, k<V ; j_{m}=0, \forall m<V \text { and } m \neq k \\
V>W . \text { Or } j_{k}>0, k=V ; j_{m}=0, \forall m<V \\
V>W . \text { Or } j_{k}>0, k=W=V .\end{array}$ \\
\hline $\begin{array}{l}\mathrm{SU}_{e}(k) \mathrm{DP} \text {. The } \mathrm{SU}_{e} \text { with the minimum number } \\
\text { of channels, } h \text {, uses all the vacant channel(s). }\end{array}$ & $\begin{array}{l}\left(i, g_{a}, j_{W}, \ldots, j_{h}-1\right. \\
\ldots, j_{k}-1, \ldots, j_{l}+1 \\
\left.\ldots, j_{V}\right)\end{array}$ & $k j_{k} \mu_{S}$ & $\begin{array}{l}j_{k}=1 ; h=\min \left\{r \mid j_{r}>0, r \in\{W, \ldots, k-1, k+1\right. \\
\ldots, V-1\}\} ; l=k+h \leq V ; V>W . \text { Or } j_{k}>1 \\
h=\min \left\{r \mid j_{r}>0, W \leq r \leq V-1\right\} ; l=k+h \leq V \\
V>W\end{array}$ \\
\hline $\begin{array}{l}\mathrm{SU}_{e}(k) \text { DP. All other } \mathrm{SU}_{e} \mathrm{~S} \text { with fewer than } V \\
\text { channels use the vacant channel(s) and achieve } \\
\text { the upper bound, } V \text {. }\end{array}$ & $\begin{array}{l}\left(i, g_{a}, 0, \ldots, 0, \ldots, 0, \ldots\right. \\
\left.j_{V}+q\right)\end{array}$ & $\begin{array}{c}\cdots \\
k j_{k} \mu_{S}\end{array}$ & $\begin{array}{l}\cdots \\
k \geq \sum_{m=1}^{V-1}(V-m) j_{m}-(V-k) ; V>W \\
q=\sum_{m=W}^{V=1} j_{m}-1\end{array}$ \\
\hline $\begin{array}{l}\mathrm{SU}_{r} \text { DP. Other } \mathrm{SU}_{e} \mathrm{~s} \text {, if exist, cannot use the } \\
\text { vacant channel(s). }\end{array}$ & $\begin{array}{l}\left(i, g_{a}-1, j_{W}, \ldots, j_{k}\right. \\
\left.\ldots, j_{V}\right)\end{array}$ & $g_{a} \mu_{S}^{\prime}$ & $\begin{array}{l}g_{a} \geq 1 ; j_{m}=0, \forall m<V ; V>W . \text { Or } g_{a} \geq 1, \\
W=V .\end{array}$ \\
\hline $\begin{array}{l}\mathrm{SU}_{r} \text { DP. The } \mathrm{SU}_{e} \text { with the minimum number } \\
\text { of channels, } h \text {, uses all the vacant channel(s). }\end{array}$ & $\begin{array}{l}\left(i, g_{a}-1, j_{W}, \ldots, j_{h}-1\right. \\
\left.\ldots, j_{l}+1, \ldots, j_{V}\right)\end{array}$ & $g_{a} \mu_{S}^{\prime}$ & $\begin{array}{l}g_{a} \geq 1 ; h=\min \left\{r \mid j_{r}>0, W \leq r \leq V-1\right\} \\
l=a+h \leq V ; V>W\end{array}$ \\
\hline $\begin{array}{l}\cdots \\
\mathrm{SU}_{r} \text { DP. All other } \mathrm{SU}_{e} \mathrm{~S} \text { with fewer than } V \\
\text { channels use the vacant channel(s) and achieve } \\
\text { the upper bound, } V \text {. }\end{array}$ & $\begin{array}{l}\left(i, g_{a}-1,0, \ldots, 0, \ldots\right. \\
\left.j_{V}+q\right)\end{array}$ & $g_{a} \mu_{S}^{\prime}$ & $\begin{array}{l}\cdots \\
g_{a} \geq 1 ; q=\sum_{m=W}^{V-1} j_{m} ; a \geq \sum_{m=W}^{V-1}(V-m) j_{m} \\
V>W\end{array}$ \\
\hline
\end{tabular}

TABLE III

TRANSITIONS FROM A GENERIC STATE $\boldsymbol{x}=\left(i, g_{a}, j_{W}, \ldots, j_{k}, \ldots, j_{V}\right)$ OF $D(a, W, V), W \leq k \leq V$ WHEN AN SU SERVICE ARRIVES.

\begin{tabular}{|c|c|c|c|}
\hline Activity & Destination state & Trans. rate & Conditions \\
\hline $\mathrm{SU}_{e}$ AR. Enough idle channels exist. & $\begin{array}{l}\left(i, g_{a}, j_{W}, \ldots, j_{k}+1, \ldots\right. \\
\left.j_{V}\right)\end{array}$ & $\lambda_{S}$ & $k=\min \{M-b(\boldsymbol{x}), V\} \geq W$ \\
\hline $\begin{array}{l}\mathrm{SU}_{e} \mathrm{AR} \text {. The } \mathrm{SU}_{e} \text { with the max- } \\
\text { imum number of channels, } m \text {, do- } \\
\text { nates channel(s) to the newly arrived } \\
\text { service. }\end{array}$ & $\begin{array}{l}\left(i, g_{a}, j_{W}+1, \ldots, j_{n}+1\right. \\
\left.\ldots, j_{m}-1, \ldots, j_{V}\right)\end{array}$ & $\lambda_{S}$ & $\begin{array}{l}V>W ; m=\max \left\{r \mid j_{r}>0, W+1 \leq r \leq V\right\} \\
n=m-[W-(M-b(\boldsymbol{x}))], W \leq n<m\end{array}$ \\
\hline $\begin{array}{l}\mathrm{SU}_{e} \mathrm{AR} \text {. Two } \mathrm{SU}_{e} \mathrm{~s}, m \text { and } h \text {, do- } \\
\text { nate channels. }\end{array}$ & $\begin{array}{l}\left(i, g_{a}, j_{W}+2, \ldots, j_{n}+1\right. \\
\ldots, j_{h}-1, \ldots, j_{m}-1, \ldots \\
\left.j_{V}\right)\end{array}$ & $\lambda_{S}$ & $\begin{array}{l}V>W>1 ; m=\max \left\{r \mid j_{r}>0, W+1 \leq r \leq V\right\} \\
h=\max \left\{r \mid j_{r}>0, W+1 \leq r \leq m-1\right\} \text { if } j_{m}=1, \text { or } \\
h=m \text { if } j_{m}>1 ; n=h+m-2 W+M-b(\boldsymbol{x}), W \leq n<h .\end{array}$ \\
\hline $\begin{array}{l}\cdots \\
\text { SU AR All SULs with more than }\end{array}$ & $\cdots$ & $\cdots$ & $\cdots=\Gamma^{V} \quad i \cdot n-\Gamma^{V}$ \\
\hline $\begin{array}{l}W \text { channels donate channel(s). } \\
W \text { ana }\end{array}$ & $\begin{array}{l}\left(\imath, g_{a}, \jmath_{W}+q, 0, \ldots, 0\right. \\
\left.j_{n}+1,0, \ldots, 0\right)\end{array}$ & $\lambda_{S}$ & $\begin{array}{l}q=\sum_{m=W+1} J_{m} ; n=\sum_{m=W+1}(m-W) J_{m}+M-b(\boldsymbol{x}) \\
W \leq n<\min \left\{r \mid j_{r}>0, W+1 \leq r \leq V\right\} ; V>W\end{array}$ \\
\hline $\mathrm{SU}_{r}$ AR. Enough idle channels exist. & $\begin{array}{l}\left(i, g_{a}+1, j_{W}, \ldots, j_{k}, \ldots\right. \\
\left.j_{V}\right)\end{array}$ & $\lambda_{S}^{\prime}$ & $M-b(\boldsymbol{x}) \geq a$ \\
\hline $\begin{array}{l}\mathrm{SU}_{r} \text { AR. The } \mathrm{SU}_{e} \text { with the max- } \\
\text { imum number of channels, } m \text {, do- } \\
\text { nates channel(s). }\end{array}$ & $\begin{array}{l}\left(i, g_{a}+1, j_{W}, \ldots, j_{n}+1\right. \\
\left.\ldots, j_{m}-1, \ldots, j_{V}\right)\end{array}$ & $\lambda_{S}^{\prime}$ & $\begin{array}{l}V>W ; m=\max \left\{r \mid j_{r}>0, W+1 \leq r \leq V\right\} \\
n=m-[a-(M-b(\boldsymbol{x}))], W \leq n<m\end{array}$ \\
\hline $\begin{array}{l}\mathrm{SU}_{r} \text { AR. Two } \mathrm{SU}_{e} \mathrm{~s}, m \text { and } h \text {, do- } \\
\text { nate channels. }\end{array}$ & $\begin{array}{l}\left(i, g_{a}+1, j_{W}+1, \ldots\right. \\
j_{n}+1, \ldots, j_{h}-1, \ldots \\
\left.j_{m}-1, \ldots, j_{V}\right)\end{array}$ & $\lambda_{S}^{\prime}$ & $\begin{array}{l}V>W ; a>1 ; m=\max \left\{r \mid j_{r}>0, W+1 \leq r \leq V\right\} \\
h=\max \left\{r \mid j_{r}>0, W+1 \leq r \leq m-1\right\} \text { if } j_{m}=1, \\
\text { or } h=m \text { if } j_{m}>1 ; n=h+m-W-a+M-b(\boldsymbol{x}), \\
W \leq n<h\end{array}$ \\
\hline $\begin{array}{l}\mathrm{SU}_{r} \text { AR. All } \mathrm{SU}_{e} \mathrm{~s} \text { with more than } \\
W \text { channels donate channel(s). }\end{array}$ & $\begin{array}{l}\left(i, g_{a}+1, j_{W}+q-1,0\right. \\
\left.\ldots, 0, j_{n}+1,0, \ldots, 0\right)\end{array}$ & $\lambda_{S}^{\prime}$ & $\begin{array}{l}\cdots \\
n=\sum_{m=W+1}^{V}(m-W) j_{m}+M-b(\boldsymbol{x})-a+W \\
W \leq n<\min \left\{r \mid j_{r}>0, W+1 \leq r \leq V\right\} ; V>W \\
q=\sum_{m=W+1}^{V} j_{m} .\end{array}$ \\
\hline
\end{tabular}

respectively, shown as follows,

$$
\mu_{p s}=\rho / \sum_{\boldsymbol{x} \in \mathcal{S}} \sum_{k=W}^{V} j_{k} \pi(\boldsymbol{x}) ; \quad \mu_{p s}^{\prime}=\rho^{\prime} / \sum_{\boldsymbol{x} \in \mathcal{S}} g_{a} \pi(\boldsymbol{x})=\mu_{S}^{\prime} .
$$

\section{B. CTMC analysis for the $D(a, W, V)$}

The feasible states in this strategy can be expressed as $\mathcal{S}=$ $\left\{\left(i, g_{a}, 0, \ldots, 0, j_{V}\right) \mid i+a g_{a}+V j_{V}<M\right\} \cup\{\boldsymbol{x} \mid b(\boldsymbol{x})=M\}$. Tables II, III, and IV summarize the state transitions in this strategy.

In a similar way as the analysis for $S(a, W, V)$, the state probability of $\pi(\boldsymbol{x})$ can be obtained and then the system performance parameters can be computed. For $D(a, W, V)$, the capacity and the service rate per ongoing SU service share the same expressions as Eqs. (2) and (4) respectively, while the blocking probability becomes,

$$
\begin{aligned}
& P_{b}=\sum_{\substack{\boldsymbol{x} \in \mathcal{S}, M-b(\boldsymbol{x})+\sum_{k=W+1}^{V}(k-W) j_{k}<W}} \pi(\boldsymbol{x}) ; \\
& P_{b}^{\prime}=\sum_{\substack{\boldsymbol{x} \in \mathcal{S}, M-b(\boldsymbol{x})+\sum_{k=W+1}^{V}(k-W) j_{k}<a}} \pi(\boldsymbol{x}) .
\end{aligned}
$$

Correspondingly, the forced termination probability is ob- 
TABLE IV

TRANSITIONS FROM A GENERIC STATE $\boldsymbol{x}=\left(i, g_{a}, j_{W}, \ldots, j_{k}, \ldots, j_{V}\right)$ OF $D(a, W, V), W \leq k \leq V$ WHEN A PU SERVICE ARRIVES.

\begin{tabular}{|c|c|c|c|}
\hline Activity & Dest. state & Trans. rate & Conditions \\
\hline PU AR. At least a vacant channel exists. & $\begin{array}{l}\left(i+1, g_{a}, j_{W}, \ldots, j_{k}\right. \\
\left.\ldots, j_{V}\right)\end{array}$ & $\lambda_{P}$ & $b(\boldsymbol{x})<M$ \\
\hline $\begin{array}{l}\mathrm{PU} \text { AR. An } \mathrm{SU}_{e}(k) \text { is interrupted and reduces } \\
\text { its channels. }\end{array}$ & $\begin{array}{l}\left(i+1, g_{a}, j_{W}, \ldots\right. \\
\left.j_{k-1}+1, j_{k}-1, \ldots, j_{V}\right)\end{array}$ & $\frac{k j_{k}}{M-i} \lambda_{P}$ & $b(\boldsymbol{x})=M ; j_{k}>0, k>W ; V>W$ \\
\hline $\begin{array}{l}\mathrm{PU} \text { AR. FT in an } \mathrm{SU}_{e}(W) \text {. No spectrum adap- } \\
\text { tation. }\end{array}$ & $\begin{array}{l}\left(i+1, g_{a}, j_{W}-1, \ldots,\right. \\
\left.j_{k}, \ldots, j_{V}\right)\end{array}$ & $\frac{W j_{W}}{M-i} \lambda_{P}$ & $\begin{array}{l}j_{W}=1 ; j_{k}=0, W+1 \leq k \leq V-1 ; b(\boldsymbol{x})=M \\
W>1 . \text { Or } j_{W} \geq 1 ; b(\boldsymbol{x})=M ; W=1 . \text { Or } \\
j_{W} \geq 1 ; b(\boldsymbol{x})=M ; W=V .\end{array}$ \\
\hline $\begin{array}{l}\mathrm{PU} \text { AR. FT in an } \mathrm{SU}_{e}(W) \text { and releases chan- } \\
\text { nel(s). The } \mathrm{SU}_{e} \text { with the minimum number of } \\
\text { channels, } h \text {, uses all the vacant channel(s). }\end{array}$ & $\begin{array}{l}\left(i+1, g_{a}, j_{W}-1, \ldots\right. \\
j_{h}-1, \ldots, j_{l}+1, \ldots \\
\left.j_{V}\right)\end{array}$ & $\frac{W j_{W}}{M-i} \lambda_{P}$ & $\begin{array}{l}j_{W}=1 ; h=\min \left\{r \mid j_{r}>0, W+1 \leq r \leq V-1\right\} \\
l=h+W-1 \leq V ; V>W>1 ; b(\boldsymbol{x})=M . \text { Or } \\
j_{W}>1 ; h=W ; l=h+W-1 \leq V ; V>W>1 ; \\
b(\boldsymbol{x})=M .\end{array}$ \\
\hline$\ldots$ & $\ldots$ & & $\ldots$ \\
\hline $\begin{array}{l}\mathrm{PU} \text { AR. FT in an } \mathrm{SU}_{e}(W) \text {. All other } \mathrm{SU}_{e} \mathrm{~s} \text { with } \\
\text { fewer than } V \text { channels use the vacant channel(s) } \\
\text { and achieve } V \text {. }\end{array}$ & $\begin{array}{l}\left(i+1, g_{a}, 0, \ldots, 0, \ldots\right. \\
\left.0, \ldots, j_{V}+q\right)\end{array}$ & $\frac{W j_{W}}{M-i} \lambda_{P}$ & $\begin{array}{l}V>W>1 ; b(\boldsymbol{x})=M ; q=\sum_{m=W}^{V-1} j_{m}-1 \\
W-1 \geq \sum_{m=W}^{V-1}(V-m) j_{m}-(V-W)\end{array}$ \\
\hline $\begin{array}{l}\mathrm{PU} \text { AR. An } \mathrm{SU}_{r} \text { is interrupted and the } \mathrm{SU}_{e} \text { with } \\
\text { the maximum number of channels, } k \text {, donates a } \\
\text { channel. }\end{array}$ & $\begin{array}{l}\left(i+1, \quad g_{a}, \quad j_{W}, \ldots\right. \\
\left.j_{k-1}+1, j_{k}-1, \ldots, j_{V}\right)\end{array}$ & $\frac{a g_{a}}{M-i} \lambda_{P}$ & $\begin{array}{l}j_{k}>0, k=\max \left\{r \mid j_{r}>0, W+1 \leq r \leq V\right\} \\
b(\boldsymbol{x})=M ; V>W\end{array}$ \\
\hline PU AR. FT in an $\mathrm{SU}_{r}$. No spectrum adaptation. & $\begin{array}{l}\left(i+1, g_{a}-1, j_{W}, \ldots\right. \\
\left.j_{k}, \ldots, j_{V}\right)\end{array}$ & $\frac{a g_{a}}{M-i} \lambda_{P}$ & $\begin{array}{l}b(\boldsymbol{x})=M ; a=1 ; g_{a} \geq 1 ; j_{r}=0, W+1 \leq r \leq V \\
\text { Or } b(\boldsymbol{x})=M ; j_{k}=0, W \leq k \leq V ; g_{a} \geq 1 \\
V>W . \text { Or } g_{a} \geq 1 ; b(\boldsymbol{x})=M ; W=V\end{array}$ \\
\hline $\begin{array}{l}\mathrm{PU} \text { AR. FT in an } \mathrm{SU}_{r} \text { and releases channel(s). } \\
\mathrm{An} \mathrm{SU}_{e}(W) \text { uses all the vacant channel(s). } \\
\ldots\end{array}$ & $\begin{array}{l}\left(i+1, g_{a}-1, j_{W}-1\right. \\
\left.\ldots, j_{k}+1, \ldots, j_{V}\right) \\
\ldots\end{array}$ & $\frac{a g_{a}}{M-i} \lambda_{P}$ & $\begin{array}{l}b(\boldsymbol{x})=M ; a>1 ; g_{a} \geq 1 ; j_{r}=0, W+1 \leq r \leq V \\
j_{W} \geq 1 ; k=a+W-1 \leq V ; V>W \\
\ldots\end{array}$ \\
\hline $\begin{array}{l}\mathrm{PU} \text { AR. FT in an } \mathrm{SU}_{r} \text {. All } \mathrm{SU}_{e} \mathrm{~s} \text { use the vacant } \\
\text { channel(s) and achieve } V \text {. }\end{array}$ & $\begin{array}{l}\left(i+1, g_{a}-1,0, \ldots, 0\right. \\
\left.\ldots, 0, \ldots, j_{V}+q\right)\end{array}$ & $\frac{a g_{a}}{M-i} \lambda_{P}$ & $\begin{array}{l}b(\boldsymbol{x})=M ; a>1 ; g_{a} \geq 1 ; j_{r}=0, W+1 \leq r \leq V \\
q=j_{W} \geq 1 ; a-1 \geq j_{W}(V-W) ; V>W\end{array}$ \\
\hline
\end{tabular}

tained as

$$
\begin{aligned}
& P_{f}=\frac{R_{f}}{\lambda_{S}^{*}}=\sum_{\substack{\boldsymbol{x} \in \mathcal{S}, b(\boldsymbol{x})=M, j_{W}>0, i<M}} \frac{\lambda_{P} W j_{W}}{(M-i) \lambda_{S}^{*}} \pi(\boldsymbol{x}) ; \\
& P_{f}^{\prime}=\frac{R_{f}^{\prime}}{\lambda_{S}^{\prime *}}=\sum_{\substack{\boldsymbol{x} \in \mathcal{S}, b(\boldsymbol{x})=M, i<M, j_{r}=0, \forall r>W}} \frac{\lambda_{P} a g_{a}}{(M-i) \lambda_{S}^{\prime *}} \pi(\boldsymbol{x}),
\end{aligned}
$$

where $\lambda_{S}^{*}=\left(1-P_{b}\right) \lambda_{S}$ and $\lambda_{S}^{\prime *}=\left(1-P_{b}^{\prime}\right) \lambda_{S}^{\prime}$.

\section{ANALYSIS IN THE QUASI-STATIONARY REGIME}

In the QSR, it is assumed that the distribution of SU services reaches equilibrium between consecutive PU events, i.e., PU activities are so slow compared with the ones of SUs that almost no forced SU termination happens ${ }^{4}$. Therefore, when $i$ PU services exist in the system, there are $M-i$ channels available and those channels are in a sense dedicated for SU services. Then, the state probability of the system can be expressed as the combination of the state probability in two Markov chains which represent PU and SU activities respectively. Let $\pi(\phi, i)$ be the state probability of the system, where $i$ denotes the number of PU services and $\phi$ represents the corresponding state for SU services, which might be multi-dimensional for a particular strategy. Then, we have $\pi(\phi, i)=\pi(\phi \mid i) \pi(i)$, where $\pi(i)$ is the state probability of having $i$ PU services, and $\pi(\phi \mid i)$ is the conditional probability

\footnotetext{
${ }^{4}$ Forced terminations will still occur but there will be, at most, one at each arrival of PU services. Since between two consecutive arrivals of PUs an infinitely large number of SU arrivals occur, the probability of forced termination is zero.
}

of state $\phi$ representing $\mathrm{SU}$ services given $i$ PU services. In the QSR, $\pi(\phi \mid i)$ can be calculated as the probability of state $\phi$ given $M-i$ channels dedicated for SUs.

In general, the state probability of PU services, $\pi(i)$, can be derived from an $M+1$ state birth and death process (BDP) with $\pi(i)=\left(\frac{\lambda_{P}}{\mu_{P}}\right)^{i} \frac{1}{i !}\left[\sum_{k=0}^{M}\left(\frac{\lambda_{P}}{\mu_{P}}\right)^{k} \frac{1}{k !}\right]^{-1}$.

To calculate $\pi(\phi \mid i)$ in this regime, we only need to consider the events of SU services given $M-i$ dedicated channels. For a given $i$, state $\phi$ contains two types of SU services, and its transitions and the corresponding rates are also illustrated in Tables I-IV, with PU events ignored. In the following, we focus on special cases when only one type of SU traffic exists in the network, in which the closed-form expressions can be further derived. We use capacity as an example but other performance parameters can also be derived in a similar way. We start from the case without real time traffic.

Proposition 1: In the QSR, the capacity of $\mathrm{SU}_{e} \mathrm{~S}$ for the dynamic strategy when real time traffic does not exist is given as follows,

$$
\rho_{e}^{d}=\left[1-\sum_{i=0}^{M} \pi(I \mid i) \pi(i)\right] \lambda_{S},
$$

where

$$
\begin{aligned}
& \pi(j \mid i)=\left\{\begin{array}{l}
\left(\frac{\lambda_{S}}{V \mu_{S}}\right)^{j} \frac{\pi(0 \mid i)}{j !}, \quad \forall 1 \leq j \leq C, \\
\left(\frac{\lambda_{S}}{Q \mu_{S}}\right)^{j} \frac{Q^{C}}{V^{C}} \frac{\pi(0 \mid i)}{C !}, \quad \forall C<j \leq I,
\end{array}\right. \\
& \pi(0 \mid i)=\left[\sum_{j=0}^{C}\left(\frac{\lambda_{S}}{V \mu_{S}}\right)^{j} \frac{1}{j !}+\sum_{j=C+1}^{I}\left(\frac{\lambda_{S}}{Q \mu_{S}}\right)^{j} \frac{Q^{C}}{V^{C} C !}\right]^{-1},
\end{aligned}
$$


and $Q=M-i, I=\left\lfloor\frac{Q}{W}\right\rfloor$, and $C=\left\lfloor\frac{Q}{V}\right\rfloor$.

The proof is deferred to Appendix A. More detailed inspection of the proof reveals that the expression for the capacity in the dynamic strategy given in Eq. (7) can actually be applied to a broader class of strategies which exhibit the following common features: 1) ongoing $\mathrm{SU}_{e} \mathrm{~S}$ will always occupy as many idle channels as they are able to; 2) if there are fewer than $W$ idle channels upon an $\mathrm{SU}_{e}$ arrival, ongoing $\mathrm{SU}_{e} \mathrm{~s}$ will donate their occupied channels to the newcomer, as long as $N \geq W$ is still satisfied for all $\mathrm{SU}_{e}$ s after donating.

Proposition 2: In the QSR, the capacity of $\mathrm{SU}_{e} \mathrm{~S}$ for the static strategy when real time traffic does not exist is upper bounded by $\rho_{e}^{s u}$,

$$
\rho_{e}^{s u}=\sum_{i=0}^{M}\left(\sum_{j=1}^{C} j V \mu_{S} \pi(j \mid i) \pi(i)+Q \mu_{S} \psi_{i} \pi(C+1 \mid i) \pi(i)\right),
$$

where $\psi_{i}=\left\lfloor\frac{Q-C V}{W}\right\rfloor$ indicates whether the state with $C+1$ services exists $\left(\psi_{i}=1\right)$ or not $\left(\psi_{i}=0\right)$ for a given $i$, and the $\pi(j \mid i)$ is calculated as follows. If $Q-C V<W, \pi(j \mid i)=$ $\left(\frac{\lambda_{S}}{V \mu_{S}}\right)^{j} \frac{1}{j !}\left[\sum_{k=0}^{C}\left(\frac{\lambda_{S}}{V \mu_{S}}\right)^{k} \frac{1}{k !}\right]^{-1}$. Otherwise, $\pi(j \mid i)$ is given by Eqs. (8) and (9) using $I=C+1$. The proof is shown in Appendix B. Note that although the closed-form capacity is not available in this strategy, we are still able to calculate its value through its CTMC model in the QSR.

Now we consider the case without elastic SU traffic and the following result is derived.

Proposition 3: In the QSR, the capacity of $\mathrm{SU}_{r} \mathrm{~s}$ when elastic traffic does not exist is,

$$
\rho_{r}=\sum_{i=0}^{M} \sum_{j=0}^{\lfloor(M-i) / a\rfloor} j \mu_{S}^{\prime} \pi(j \mid i) \pi(i),
$$

where $\pi(j \mid i)=\left(\frac{\lambda_{S}^{\prime}}{\mu_{S}^{\prime}}\right)^{j} \frac{1}{j !}\left[\sum_{k=0}^{\lfloor(M-i) / a\rfloor}\left(\frac{\lambda_{S}^{\prime}}{\mu_{S}^{\prime}}\right)^{k} \frac{1}{k !}\right]^{-1}$.

The mathematical proof of this result is similar to Proposition 1 when $W=V=a$. Hence, we do not show the detailed proof to save space.

\section{NUMERICAL RESULTS AND DISCUSSIONS}

In this section, the obtained numerical results under different conditions are presented. In the first subsection, the performance of the investigated strategies with pure elastic SU traffic is illustrated. In the second subsection, we observe the results when both types of SU traffic co-exist. Furthermore, the results in the QSR are plotted in comparison with the results from the original CTMC models in the third subsection, and the results under diverse traffic distributions are illustrated in the final subsection.

In order to verify the mathematical models, the simulation results obtained from a custom-built MATLAB simulator together with the analytical results are plotted in Figs. 1-5. In these figures, the solid lines represent the analytical results while the marks are obtained from simulations. From these curves, we can observe that the simulation results precisely coincide with the analytical results. Therefore, the correctness of the mathematical analysis is validated.

The simulations are conducted as follows. Each round of the simulation is performed for 10000 time units. Each time unit, denoted as $T$, is further divided into $n$ sub-intervals each with duration $h$, i.e., $T=n h$, where $h$ is the minimum unit in this simulation and it is configured sufficiently small so that the probability of simultaneous events is ignorable. Then the probability that exactly one $\mathrm{PU}, \mathrm{SU}_{e}$, or $\mathrm{SU}_{r}$ arrival occurs in each sub-interval is $\lambda_{P} h, \lambda_{S} h$, or $\lambda_{S}^{\prime} h$, and the probability of no arrival is $1-\lambda_{P} h, 1-\lambda_{S} h$, or $1-\lambda_{S}^{\prime} h$ respectively. The lengths of PU and SU services are integer times of $h$, following exponential distributions with parameters corresponding to various services. For an ongoing PU service, after each $h$, the integer will decrease by one, meaning that part of this PU service has elapsed. The same principle applies to $\mathrm{SU}_{r}$. However, for an ongoing $\mathrm{SU}_{e}$ with $N$ channels, after each $h$, the integer will decrease by $N$ since the service rate will increase $N$ times. In each sub-interval, the events of both PUs and SUs are checked and processed according to the employed strategy. The service is considered as finished when this integer number reaches zero. In the end of each simulation, the statistics of the system can be calculated. For example, the capacity is obtained from the total number of successful SU services averaged by the total simulated time units.

\section{A. Performance illustration with pure elastic SU traffic}

Since only $\mathrm{SU}_{e} \mathrm{~S}$ will contribute to the system performance with channel assembling, we examine the cases where only $\mathrm{SU}_{e} \mathrm{~s}$ exist in the secondary network first. In this subsection, $\rho, P_{b}, P_{f}$, and $\mu_{p s}$ are plotted in Figs. 1-4 respectively, given $M=6, \lambda_{S}^{\prime}=0, \lambda_{S}=1.5, \mu_{S}=0.82$, and $\mu_{P}=0.5$. In the labels of strategies in these figures, $a$ is not given a specific value since $\mathrm{SU}_{r} \mathrm{~s}$ do not exist. In order to compare the effects of different threshold values, we plot two groups of results for each strategy, i.e., $W=1, V=3$ and $W=3, V=6$. The results of $N A$ are also illustrated for the comparison purpose.

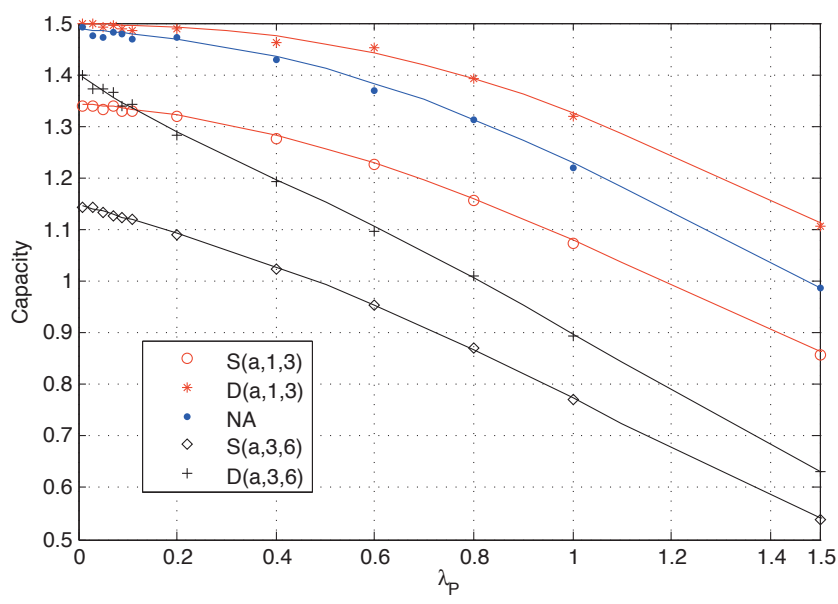

Fig. 1. System capacity as a function of $\lambda_{P}$ for pure elastic SU traffic.

1) System capacity: As shown in Fig. 1, the system capacity of $\mathrm{SU}_{e} \mathrm{~S}$ decreases for all strategies with an increasing $\lambda_{P}$ due to the fact that SUs get fewer chances to start their services when PUs become more active. Interestingly, most of the channel assembling strategies do not perform better than $N A$ does, except $D(a, 1,3)$. The advantage of $N A$ is because that a larger number of parallel services, i.e., $j_{k}$ 


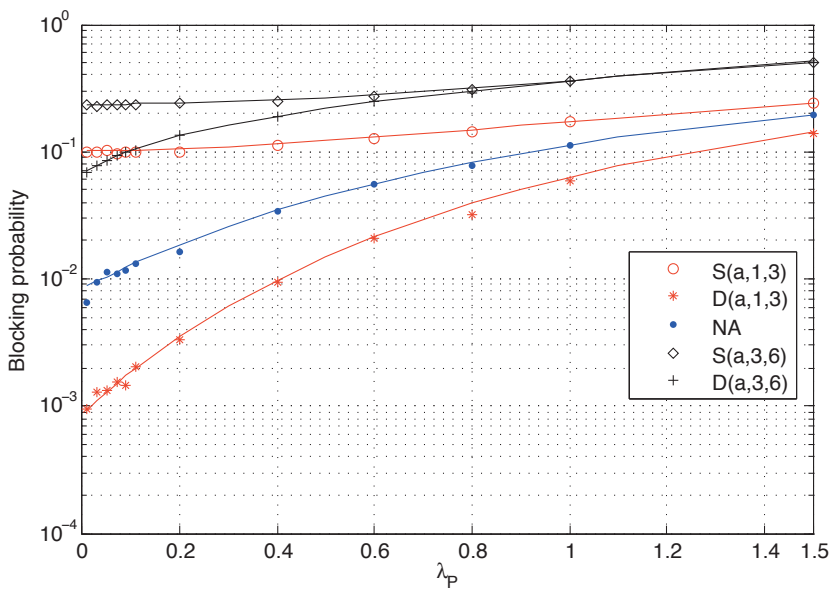

Fig. 2. Blocking probability as a function of $\lambda_{P}$ for pure elastic SU traffic.

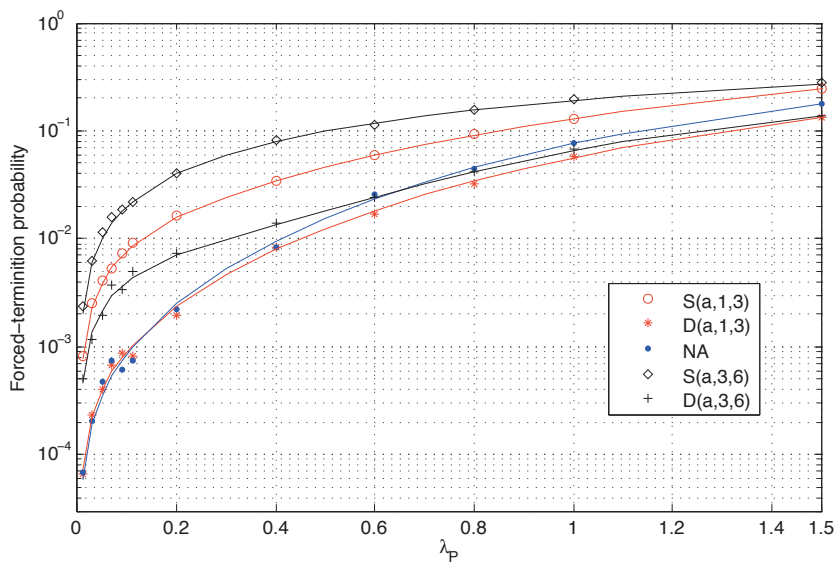

Fig. 3. Forced termination probability as a function of $\lambda_{P}$ for pure elastic SU traffic.

in Eq. (2), could contribute to system capacity significantly. Dynamic strategies achieve higher capacity than static ones with the same parameter configuration. Comparing two groups of $W=1, V=3$ with $W=3, V=6$, the results in the former group for each strategy are better than the corresponding latter ones. The reason is that in the latter group, the strategies require at least three vacant channels out of a total number of six channels in order to start an SU service, leading to wasted spectrum opportunities compared with the strategies in the former group.

2) Blocking probability: Fig. 2 depicts the blocking probability of SUs. One can observe that $D(a, 1,3)$ has the lowest blocking probability since it needs only one channel for initiating an SU service and can dynamically adjust the number of assembled channels upon different events. Similarly, $N A$ has the second lowest blocking probability as compared with the other strategies. Comparing the group $W=1, V=3$ with $W=3, V=6$, the blocking probability is generally higher in the latter group. The reason is straightforward since more channels are required in the latter case before a service request can be accepted.

3) Forced termination probability: To examine the forced termination probability of the commenced SU services, we plot $P_{f}$ in Fig. 3. As expected, $P_{f}$ becomes higher for all strategies

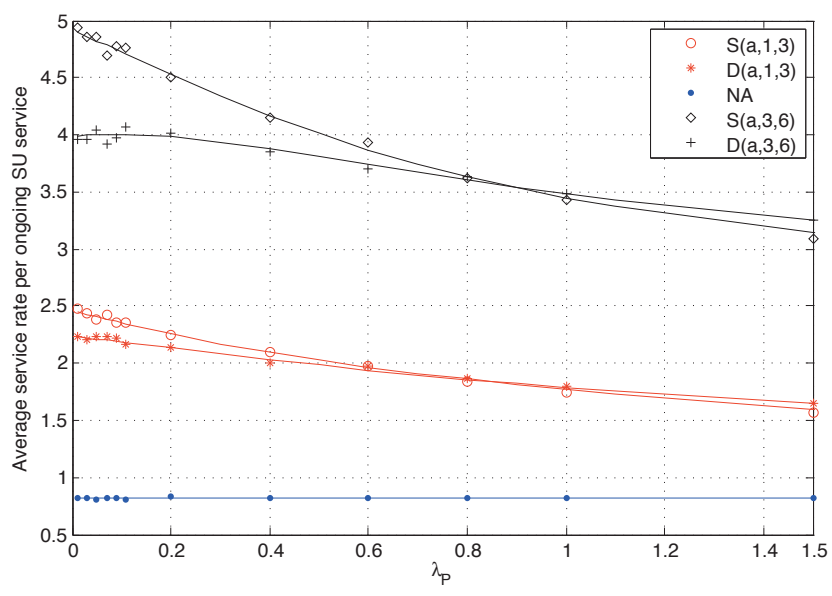

Fig. 4. Average service rate per ongoing SU service as a function of $\lambda_{P}$ for pure elastic SU traffic.

as $\lambda_{P}$ increases since PUs become more active. The forced termination probability in $N A$ is not as good as that of the $D(a, 1,3)$, and increases even higher than that of $D(a, 3,6)$ when $\lambda_{P}$ is large. It means that a lower $P_{f}$ could be achieved if an appropriate strategy and suitable threshold values are selected. For the static strategies, since they will assemble multiple channels and the number of assembled channels is not adjustable, services would be more likely forced to terminate than the dynamic cases.

4) Average service rate per ongoing SU service: Fig. 4 illustrates the average service rate per ongoing SU service, i.e., $\mu_{p s}$. This value indicates the rate that an ongoing SU service will be processed on average. As illustrated in this figure, the larger the number of channels an SU service assembles, the higher the average service rate per ongoing service a strategy can achieve. For $N A$, the $\mu_{p s}$ value does not change with different $\lambda_{P}$ values since each SU service occupies only one channel all the time thus $\mu_{p s}=\mu_{S}$, whereas the other curves decline as $\lambda_{P}$ increases.

It is worth mentioning that $\mu_{p s}$ shown in Fig. 4 is the average service rate per ongoing SU service, which is different from the total average service rate for SU services, i.e., capacity, $\rho$, shown in Fig. 1. As can be observed from Eq. (4), $\mu_{p s}$ is equal to the capacity value, $\rho$, divided by the average number of ongoing SU services, meaning that a strategy with a larger $\mu_{p s}$ value does not necessarily lead to higher capacity. For example, $S(a, 3,6)$ has the highest $\mu_{p s}$ however the lowest capacity when $\lambda_{P}$ is small as observed in Fig. 1. The reason is that with a small $\lambda_{P}$ the forced termination probability and the blocking probability for this strategy are higher than those of other strategies. Indeed we observe in our simulations a much lower average number of ongoing SU services in the system with this strategy, even though each ongoing SU service occupies more channels.

\section{B. Performance illustration with heterogenous traffic}

In Fig. 5, it is illustrated the achieved capacity of the strategies in presents of heterogenous traffic. We adopt $a=1$, $\lambda_{S}^{\prime}=1$ and $\mu_{S}^{\prime}=0.6$ for the real time traffic and utilize 


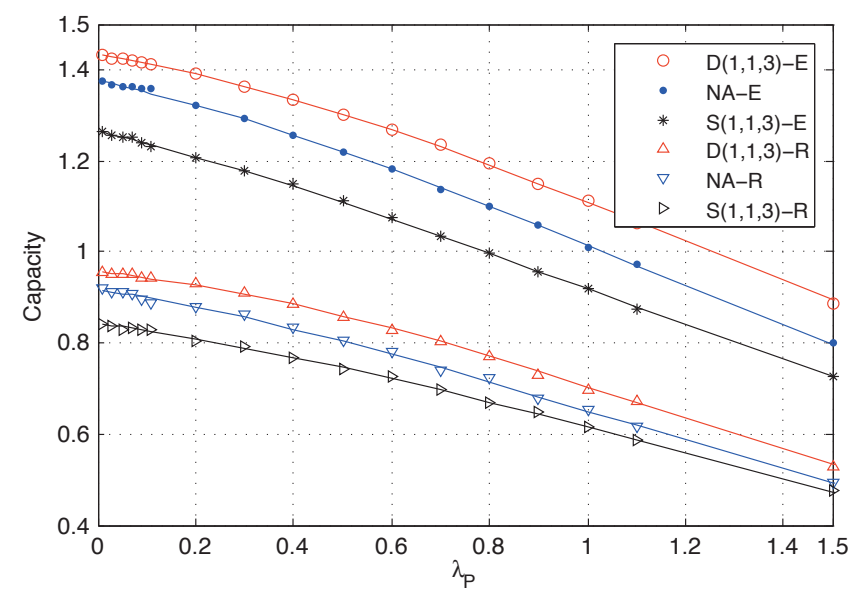

Fig. 5. Capacity as a function of $\lambda_{P}$ for heterogeneous traffic. In this figure, $E$ indicates elastic traffic while $R$ denotes real time traffic.

the same parameter for $\mathrm{PUs}$ and $\mathrm{SU}_{e} \mathrm{~s}$ as in the previous subsection. From Fig. 5, one can observe that the capacity for $\mathrm{SU}_{e} \mathrm{~s}$ of all these strategies is lower than the one shown in Fig. 1 because the resource is shared with real time traffic. Compared with $N A$, the dynamic strategy achieves higher capacity in both $\mathrm{SU}_{e}$ and $\mathrm{SU}_{r}$, which means that the advantage of channel assembling in $\mathrm{SU}_{e} \mathrm{~S}$ is shared among these two traffic types. In contrast, the static ones cannot provide any benefit in terms of capacity. The advantage of the dynamic channel assembling strategy is also observed in all other performance parameters however not shown here due to page limit. The main observation here is that the benefit of channel assembling using the dynamic strategy is obvious and the advantage obtained by $\mathrm{SU}_{e}$ is shared with real-time flows. However, the static strategy has poorer performance than $N A$ except when $\mu_{p s}$ is investigated.

\section{Performance in the QSR}

In Fig. 6, the results from the analytical models in the QSR and those from the precise models developed in Section V, are illustrated. In this subsection, we introduce a scaler, $f$, to reflect the dynamics of PU activities while keeping the offered load constant for both PU and SU services, as $\lambda_{P}=1 \times f$, $\mu_{P}=0.5 \times f, \lambda_{S}^{\prime}=0, \lambda_{S}=1.5, \mu_{S}=0.82$ and $M=6$.

As illustrated in Fig. 6, when $f<<1$, meaning that the service time of PUs is quite long while the arrival rate is low, i.e., PUs appear sporadically and PU services are longlasting, the system capacity deduced from the precise models fits the results in the QSR well. When $f$ becomes larger, as $1<f<10^{3}$, meaning that PUs are more dynamic, the capacity obtained from the precise models deviates more and more from the corresponding results in the QSR, due to the fact that more and more SU connections are abruptly terminated by PUs. Moreover, when $f$ becomes even larger as $f>10^{4}$, the capacity is close to zero for all the investigated strategies, since PUs are so active that SU services cannot survive from preemption.

Not surprisingly, the dynamic strategy has again achieved the best performance when comparing the results in the

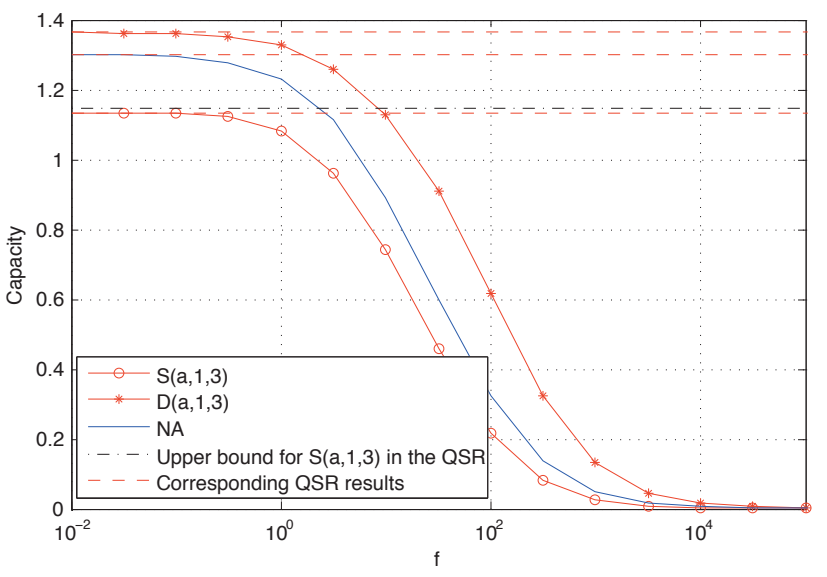

Fig. 6. System capacity as a function of $f$. The dynamic behavior of PU activities is represented by a traffic intensity scale $f$. The results in the QSR of $D(a, 1,3)$ is calculated based on Eq. (7), and that of $N A$ is also achieved by the same equation given $V=W=1$. The upper bound for $S(a, 1,3)$ in the QSR is plotted according to Eq. (10), while its precise capacity in the QSR is calculated through its CTMC model in this regime.

QSR among different strategies, followed by the one without channel assembling. Moreover, the static strategy has lower capacity than its upper bound given by Eq. (10) in the QSR, confirming Proposition 2.

\section{Traffic pattern with various distributions}

In our previous discussions, Poisson arrival and exponentially distributed service time are assumed. In real life, however, the traffic patterns might be quite different from those ones. This observation triggers our motivation to further investigate the applicability and the preciseness of our Markov chain based analytical models, by applying the same simulation programs mentioned earlier to diverse PU and SU traffic distributions.

As an example, Fig. 7 illustrates the forced termination probability of two sample strategies with pure elastic SU traffic, i.e., $N A$ and $D(a, 1,3)$ as a function of $\lambda_{S}$, under two traffic models based on real-life traffic observations [19], [20]. For traffic pattern one, Poisson arrival and log normal distributed service time for both PU and SU services are utilized. Within this traffic type, we consider further two cases. The first case is that both the mean values and the variances of the $\log$ normal distributions equal to those of the corresponding original exponential distributions, labeled as $L N$. The other case is that the variance values of log normal distributions are greater than those of the original exponential distributions while the mean values are kept the same, labeled as $L N^{*}$. The reason for using larger variance values in $L N^{*}$ is that it can model more precisely the traffic in modern data networks where high variability has been observed in flow sizes [20]. More specifically, we adopt a squared coefficient of variation $\mathrm{SCV}=4.618$ [20] $\left(\mathrm{SCV}=\right.$ variance $\left./ \mathrm{mean}^{2}\right)$. For traffic pattern two, a random walk model for PU activities [19], and Poisson arrival and $\log$ normal distributed service time for SU services are adopted, labeled as $R W$. For the $\log$ normal distribution of SU service time used in this case, we make the mean value and the variance equal to those of the 


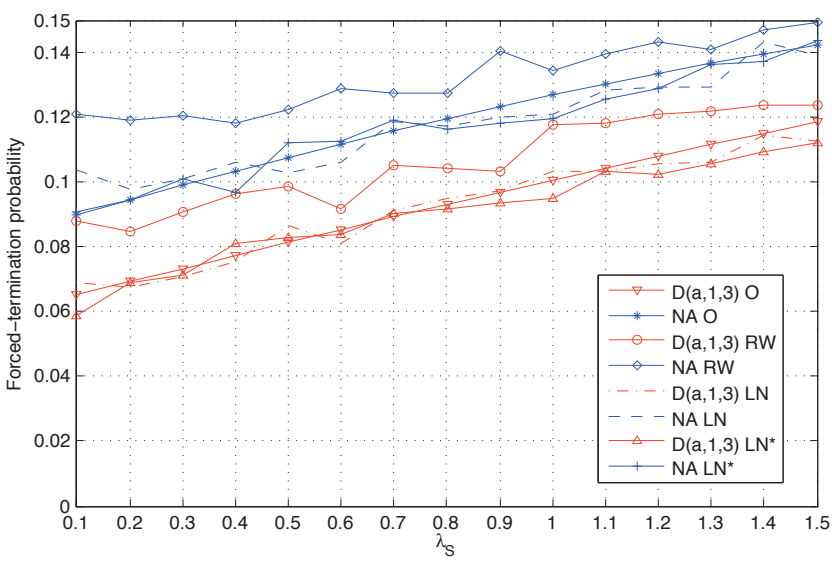

Fig. 7. Forced termination probability as a function of $\lambda_{S}$ when various distributions are utilized.

original exponential distribution. The results from the original distributions in our mathematical models are also plotted for comparison purpose, labeled as $O$.

From this figure, we can observe that the results of $L N$ and $L N^{*}$ are quite close to the analytical ones, which reveals the fact that these results are not sensitive to the distribution type of service time as long as Poisson arrival is kept. Moreover, although the results under $R W$ do not coincide with the analytical results precisely, those curves still exhibit similar results to a large extent. Other simulation results with different parameter configurations, even though not explicitly illustrated here, yield also similar results. Therefore, the results under different traffic models are comparatively close to the ones obtained based on Poisson arrival and exponential service time distributions. These observations demonstrate that although different distributions exist, the mathematical analysis presented in this paper can be adopted as a robust reference model for analyzing the performance of channel assembling strategies in CRNs.

In more details, Fig. 7 are obtained as follows. There are altogether $M=6$ channels and the SU service rate is $\mu_{S}=$ 0.5 in the original exponential service time distribution. For an equal comparison, we set $\lambda_{P}=0.5$ and $\mu_{P}=0.15601$ in the original distributions for PUs. This configuration gives three PU-occupied channels in the system on average, which is equal to the average number of PU-occupied channels in the random walk model [19]. Then the average time interval between two PU events in the random walk model, $\tau$, is computed as $\tau=$ $1 /\left(2 \mu_{P} E\right)=1.0683$, where $E=3$ is the average number of PU-occupied channels.

\section{E. Further discussions}

Consider channel allocation from a contrary perspective in which an SU service requires less than one channel, i.e., one or several sub-channels within one ordinary channel. In this case, known as channel splitting, more SU services can be accommodated in a system given the assumption that $\mathrm{SU}$ services may be provided based on one or multiple subchannels. In the QSR, since idle channels are in a sense dedicated for SUs, the same analysis as illustrated in Section
VI can be applied to channel splitting on a sub-channel basis. However, without the QSR assumption, the arrival of one PU service may influence multiple SU services and the analysis will then become more sophisticated.

Finally, to make our channel assembling strategies smarter, the lower and upper bounds, i.e., $W$ and $V$, can be adjusted dynamically according to different parameters. For example, the value of $V$ in $D(a, 1,3)$ can be tuned according to the arrival rate of PUs. As shown in Fig. 1, when $\lambda_{P}$ is less than 0.1 , the performance of $N A$ and $D(a, 1,3)$ is quite close to each other. Therefore, $V=1$, i.e., $N A$ is recommended since it can achieve similar capacity without employing complicated channel assembling algorithm. When $\lambda_{P}$ becomes larger, however, $V=3$ will be selected in order to achieve better performance. In real life, a measurement-based estimation of the arrival intensities can be utilized to feed our analytical models for parameter calculations so that $W$ and $V$ can be adjusted accordingly on the fly.

\section{CONCLUSIONS}

In this paper, channel assembling approaches represented by the two proposed strategies in multi-channel CRNs are investigated thoroughly considering heterogeneous SU traffic types, and their performance is evaluated and compared with each other and with the legacy no assembling strategy through both mathematical analyses and simulations. The numerical results demonstrate that the dynamic strategy can achieve better performance than the strategy without assembling does in the investigated cases. For the static strategy, however, its overall performance is poorer than the no channel assembling case except one aspect, i.e., a higher average service rate per ongoing SU service. Therefore, we would recommend the dynamic strategy as the most appropriate alternative for channel assembling in multi-channel CRNs.

\section{APPENDIX A \\ PROOF OF PROPOSITION 1}

Proof: Without $\mathrm{SU}_{r} \mathrm{~s}, g_{a}$ can be eliminated from $\phi$. However, $\phi=\left(j_{W}, \ldots, j_{V}\right)$ may still be multi-dimensional for a given $i$. In what follows, we will firstly map the process for SU services from a multi-dimensional Markov chain into a BDP as shown in Fig. 8 for a given $i$, and then calculate the capacity based on the BDP.

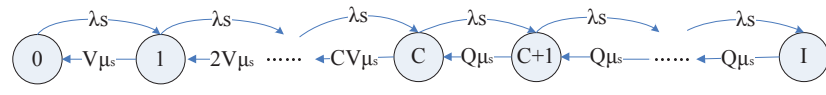

Fig. 8. The birth and death process of SU services for a given $i$.

Consider all states of SU services for a given $i$. Let us rearrange these states according to the number of ongoing SU services that these states have, i.e., using an integer pair $(r, l)$ to represent a state, where $r$ denotes the number of ongoing SU services and $l$ represents a particular state among all the states with $r$ SU services. Let $L(r)$ be the number of states that have $r$ SU services, therefore we have $l \in\{1, \ldots, L(r)\}$. Let $\pi^{\prime}(r, l)$ be the corresponding state probability. Denote $|\phi|$ as 


$$
\begin{aligned}
& \mu_{S}\left(m(r)-\frac{\sum_{l=1}^{L(r)}(m(r)-b(r, l)) \pi^{\prime}(r, l)}{\sum_{l=1}^{L(r)} \pi^{\prime}(r, l)}\right) \sum_{l=1}^{L(r)} \pi^{\prime}(r, l)+\lambda_{S} \sum_{l=1}^{L(r)} \pi^{\prime}(r, l) \\
& =\lambda_{S} \sum_{n=1}^{L(r-1)} \pi^{\prime}(r-1, n)+\mu_{S}\left(m(r+1)-\frac{\sum_{l=1}^{L(r+1)}(m(r+1)-b(r+1, l)) \pi^{\prime}(r+1, l)}{\sum_{l=1}^{L(r+1)} \pi^{\prime}(r+1, l)}\right) \sum_{l=1}^{L(r+1)} \pi^{\prime}(r+1, l) .
\end{aligned}
$$

the number of ongoing SU services at state $\phi$. For a generic state $\phi$, say, the lth state with $|\phi|=r$ SU services, where $r \in\{1, \ldots C-1\}$, the balance equation can be expressed as:

$$
\begin{aligned}
& \left(\lambda_{S}+r V \mu_{S}\right) \pi^{\prime}(r, l)=\sum_{n=1}^{L(r-1)} P_{r-1, n, l} \lambda_{S} \pi^{\prime}(r-1, n) \\
& +\sum_{n=1}^{L(r+1)} P_{r+1, n, l}^{\prime}(r+1) V \mu_{S} \pi^{\prime}(r+1, n)
\end{aligned}
$$

where $P_{r-1, n, l}$ is the probability from state $(r-1, n)$ to state $(r, l)$ upon an SU arrival while $P_{r+1, n, l}^{\prime}$ is the probability from state $(r+1, n)$ to state $(r, l)$ upon an SU departure. $P_{r-1, n, l}$ and $P_{r+1, n, l}^{\prime}$ represent different ways of access upon an event in this strategy. Note that $\sum_{m=1}^{L(r)} P_{r-1, n, m}=1$ and $\sum_{m=1}^{L(r)} P_{r+1, n, m}^{\prime}=1$. If we sum up all these equations of states with $r$ SU services, the left hand side can be expressed as a common factor of outgoing rates by the sum of the probabilities of the states with $r$ SU services. Similarly, on the right hand side, the transitions indicating SU arrivals share the common factor $\lambda_{S}$ while the transitions indicating SU departures share the same $r V \mu_{S}$. Consequently, the following equation holds,

$$
\begin{aligned}
& \left(\lambda_{S}+r V \mu_{S}\right) \sum_{l=1}^{L(r)} \pi^{\prime}(r, l)=\lambda_{S} \sum_{n=1}^{L(r-1)} \pi^{\prime}(r-1, n) \\
& +(r+1) V \mu_{S} \sum_{n=1}^{L(r+1)} \pi^{\prime}(r+1, n) .
\end{aligned}
$$

If we regard the sum of the probabilities of all states satisfying $|\phi|=r$ as a whole, Eq. (14) is the same as the balance equation for the state with $r$ services in the BDP as shown in Fig. 8. Therefore, the sum of the probabilities of all states with $|\phi|=r$ can be regarded as one state probability with $r$ services in the corresponding BDP. Similar analysis applies to $r \in\{C, \ldots I\}$. To calculate the capacity, knowing the sum of the probability of the states that have the same number of SU services given $i$ PU services is sufficient since the service rates are identical for all these states.

The state probability for the $\mathrm{BDP}$, for a given $i$, is derived as shown in Eqs. (8) and (9). Considering all possible $i$, the blocking probability for SU services becomes $P_{b}=$ $\sum_{i=0}^{M} \pi(I \mid i) \pi(i)$. Since capacity can be represented by the completed SU services over time, i.e., the commenced and survived (not forced to terminate) ones among all arrivals, we have $\rho=\left(1-P_{b}\right)\left(1-P_{f}\right) \lambda_{S}$. Considering $P_{f}=0$ in this regime, the capacity can be expressed as shown in Eq. (7).

\section{APPENDIX B \\ PROOF OF PROPOSITION 2}

Proof: Without $\mathrm{SU}_{r} \mathrm{~s}, g_{a}$ can be eliminated from $\phi$. Therefore, $\phi=\left(j_{W}, \ldots, j_{V}\right)$. In the static strategy, since ongoing SU services cannot adjust the number of their channels, the states that have the same number of ongoing $\mathrm{SU}$ services may have different assembled service rates given $i$ PU services. Let us label all states satisfying $|\phi|=r$ in two dimensions, as $(r, l)$, indicating the $l$ th state which has $r$ SU services. For a generic state $(r, l)$, denote $b(r, l)$ as the number of channels that all ongoing SU services assemble at state $(r, l)$, i.e., $b(r, l)=\sum_{k=W}^{V} k j_{k}$ for $(r, l) \Leftrightarrow\left(j_{W}, \ldots, j_{k}, \ldots, j_{V}\right)$. Then the balance equation for $(r, l)$ can be expressed as:

$$
\begin{aligned}
& \left(\lambda_{S}+b(r, l) \mu_{S}\right) \pi^{\prime}(r, l)=\sum_{n=1}^{L(r-1)} P_{r-1, n, l} \lambda_{S} \pi^{\prime}(r-1, n) \\
& +\sum_{n=1}^{L(r+1)} P_{r+1, n, l}^{\prime} b(r+1, n) \mu_{S} \pi^{\prime}(r+1, n)
\end{aligned}
$$

where $\pi^{\prime}(r, l), L(r), P_{r-1, n, l}$, and $P_{r+1, n, l}^{\prime}$ have the same definition as in Appendix A.

Let $m(r)=\max _{l}(b(r, l))$. Summing up all the balance equations for states with $|\phi|=r$, we have Eq. (12). Look at the service rate for states with $r$ services on the left hand side of Eq. (12). Clearly, $m(r) \leq r V$, i.e., $m(r)$ is less than or equal to the maximum number that $r$ SU services can utilize. Note that $\frac{\sum_{l=1}^{L(r)}(m(r)-b(r, l)) \pi^{\prime}(r, l)}{\sum_{l=1}^{L(r)} \pi^{\prime}(r, l)}$ is non-negative. Therefore $\left(m(r)-\frac{\sum_{l=1}^{L(r)}(m(r)-b(r, l)) \pi^{\prime}(r, l)}{\sum_{l=1}^{L(r)} \pi^{\prime}(r, l)}\right) \leq r V$. The service rate on the right hand side of the equation, i.e., the one corresponds to states with $r+1$ services, follows the same principle. Consequently, for the state with $r$ SU services in the BDP based on the static strategy, the service rate will not be higher than the corresponding one that will always achieve the maximum possible service rate. If we make the service rate for all states with $r$ services equaling to the maximum possible service rate, i.e., let $b(r, l)=m(r)$, the capacity can be derived in a closed form as shown in Eq. (10). With a lower or an equal service rate in each state for the static strategy than the one shown in Eq. (10), the capacity, i.e., the average number of SUs that can be served at a time unit, will be upper bounded by $\rho_{e}^{s u}$.

\section{REFERENCES}

[1] Y. -C. Liang, K. -C. Chen, G. Y. Li, and P. Mähönen, "Cognitive radio networking and communications: An overview", IEEE Trans. Veh. Technol., vol. 60, no. 7, pp. 3386-3407, Sep. 2011. 
[2] B. Hamdaoui and K. G. Shin, "OS-MAC: An efficient MAC protocol for spectrum-agile wireless networks," IEEE Trans. Mobile Comput., vol. 7, no. 8, pp. 915-930, Aug. 2008.

[3] Q. Chen, Y. -C. Liang, M. Motani, and W. -C. Wong, "A two-level MAC protocol strategy for opportunistic spectrum access in cognitive radio networks", IEEE Trans. Veh. Technol., vol. 60, no. 5, pp. 1-23, Jun. 2011.

[4] S. Huang, X. Liu, and Z. Ding, "Optimal transmission strategies for dynamic spectrum access in cognitive radio networks," IEEE Trans. Mobile Comput., vol. 8, no. 12, pp. 1636-1648, Nov. 2009.

[5] H. Su and X. Zhang, "Channel-hopping based single transceiver MAC for cognitive radio networks," in Proc. IEEE CISS, Princeton, NJ, USA, Mar. 2008, pp. 197-202.

[6] H. Su and X. Zhang, "Cross-Layer based opportunistic MAC protocols for QoS provisionings over cognitive radio mobile wireless networks," IEEE J. Select. Areas Commun., vol. 26, no. 1, pp. 118-129, Jan. 2008.

[7] Y. Yuan, P. Bahl, R. Chandra, P. A. Chou, J. I. Ferrell, T. Moscibroda, S. Narlanka and Y. Wu, "KNOWS: Cognitive radio networks over white spaces," in Proc. IEEE DySPAN, Dublin, Ireland, Apr. 2007, pp. 416-427.

[8] IEEE 802.22 WG, IEEE Standard for Wireless Regional Area Networks Part 22: Cognitive Wireless RAN Medium Access Control (MAC) and Physical Layer (PHY) Specifications: Policies and Procedures for Operation in the TV Bands, IEEE Standard, July 2011.

[9] J. Jia, Q. Zhang, and X. Shen, "HC-MAC: A hardware-constrained cognitive MAC for efficient spectrum management," IEEE J. Select. Areas Commun., vol. 26, no. 1, pp. 106-117, Jan. 2008.

[10] H. A. B. Salameh, M. M. Krunz, and O. Younis, "MAC protocol for opportunistic cognitive radio networks with soft guarantees," IEEE Trans. Mobile Comput., vol. 8, no. 10, pp. 1339-1352, Oct. 2009.

[11] A. C. Hsu, D. S. L. Wei, and C. -C. J. Kuo, "A cognitive MAC protocol using statistical channel allocation for wireless ad-hoc networks," in Proc. IEEE WCNC, Hong Kong, China, Mar. 2007, pp. 105-110.

[12] P. K. Tang, and Y. H. Chew, "On the modeling and performance of three opportunistic spectrum access schemes," IEEE Trans. Veh. Technol., vol. 59, no. 8, pp. 4070-4078, Oct. 2010.

[13] X. R. Zhu, L. F. Shen, and T. -S. P. Yum, "Analysis of cognitive radio spectrum access with optimal channel reservation," IEEE Commun. Lett., vol. 11, no. 4, pp. 304-306, Apr. 2007.

[14] J. Martinez-Bauset, V. Pla, and D. Pacheco-Paramo, "Comments on 'Analysis of cognitive radio spectrum access with optimal channel reservation'," IEEE Commun. Lett., vol. 13, no. 10, pp. 739, Oct. 2009.

[15] E. W. M. Wong, and C. H. Foh, "Analysis of cognitive radio spectrum access with finite user population," IEEE Commun. Lett., vol. 13, no. 5, pp. 294-296, May 2009.

[16] L. Jiao, V. Pla, and F. Y. Li, "Analysis on channel bonding/aggregation for multi-channel cognitive radio network," in Proc. European Wireless, Lucca, Italy, Apr. 2010, pp. 468-474.

[17] J. Lee, and J. So, "Analysis of cognitive radio networks with channel aggregation," in Proc. IEEE WCNC, Sydney, Australia, Apr. 2010, pp. 1-6.

[18] L. Jiao, F. Y. Li, and V. Pla, "Dynamic channel aggregation strategies in cognitive radio networks with spectrum adaptation," in Proc. IEEE GLOBECOM, Houston, TX, USA, Dec. 2011, pp. 1-6.

[19] D. Willkomm, S. Machiraju, J. Bolot, and A. Wolisz, "Primary users behavior in cellular networks and implications for dynamic spectrum access," IEEE Commun. Mag., vol. 47, no. 3, pp. 88-95, Mar. 2009.

[20] P. Barford and M. Crovella, "Generating representative Web workloads for network and server performance evaluation," in Proc. ACM SIGMETRICS, Madison, WI, USA, Jul. 1998, pp. 151-160.

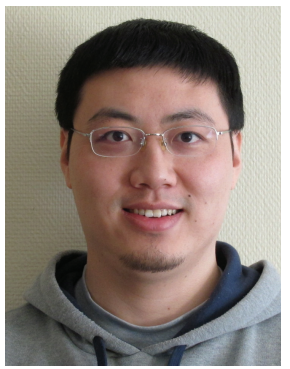

Lei Jiao (S'08) received his BE and ME degrees in Telecommunication Engineering, and Communication and Information System from Hunan University and Shandong University, China respectively in 2005 and 2008. Since August 2008, he is with the Department of Information and Communication Technology, University of Agder (UiA), Norway, pursuing his $\mathrm{Ph}$. D. degree. His research interests include cognitive radio networks, wireless sensor networks, localization algorithms and intelligent transportation systems.

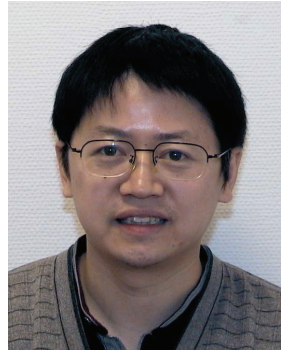

Frank Y. Li (S'99-M'03-SM'09) holds a Ph.D. degree from the Norwegian University of Science and Technology (NTNU). He worked as a Senior Researcher at UniK - University Graduate Center, University of Oslo before joining the Department of Information and Communication Technology, University of Agder (UiA) in August 2007 where he is currently a Professor. During the past few years, he has been an active participant in several Norwegian and EU FP6/FP7 research projects. He is listed as a Lead Scientist by the European Commission DG RTD Unit A.03 - Evaluation and Monitoring of Programmes in Nov. 2007. Dr. Li's research interests include $3 \mathrm{G} / 4 \mathrm{G}$ and beyond mobile systems and wireless networks, mesh and ad hoc networks; wireless sensor network; cooperative communications; cognitive radio networks; green wireless communications; QoS, resource management and traffic engineering in wired and wireless IPbased networks; analysis, simulation and performance evaluation of communication protocols and networks.

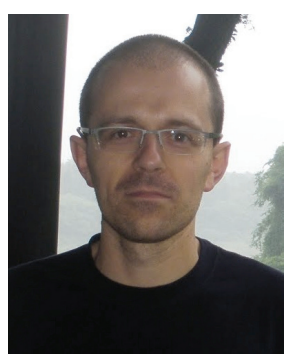

Vicent Pla obtained his Engineering degree and $\mathrm{Ph} . \mathrm{D}$. degree in Telecommunication Engineering from the Universitat Politècnica de València (UPV), Spain. Currently, he is an Associate Professor at the Department of Communications at UPV. His research interests lie primarily in the areas of teletraffic, modeling and performance evaluation of communication networks. In the past years most of his research activity has focused on the area of resource management in wireless networks. In these areas he has published numerous papers in refereed journals and conference proceedings. 